\title{
Comparison of tests for embeddings
}

\author{
C. Letellier, ${ }^{1}$ I. M. Moroz, ${ }^{2}$ and R. Gilmore ${ }^{1,3}$ \\ ${ }^{1}$ Université de Rouen, CORIA UMR 6614, BP 12, F-76801 Saint-Etienne du Rouvray cedex, France \\ ${ }^{2}$ Mathematical Institute, 24-29 St Giles', Oxford OX1 3LB, United Kingdom \\ ${ }^{3}$ Physics Department, Drexel University, Philadelphia, Pennsylvania 19104, USA
}

(Received 17 December 2007; published 6 August 2008)

\begin{abstract}
It is possible to compare results for the classical tests for embeddings of chaotic data with the results of a recently proposed test. The classical tests, which depend on real numbers (fractal dimensions, Lyapunov exponents) averaged over an attractor, are compared with a topological test that depends on integers. The comparison can only be done for mappings into three dimensions. We find that the classical tests fail to predict when a mapping is an embedding and when it is not. We point out the reasons for this failure, which are not restricted to three dimensions.
\end{abstract}

DOI: 10.1103/PhysRevE.78.026203

PACS number(s): 05.45.-a

\section{INTRODUCTION}

The treatment of data generated by chaotic dynamical systems involves two separate steps. The first is the search for an embedding of the data into a phase space of suitable dimension. The second is an analysis of the reconstructed chaotic attractor once a suitable embedding has been found.

At the analysis stage, the embedded attractor can be studied using tools that can broadly be classified as geometric, dynamical, or topological. The geometric and dynamical tools can be applied to an attractor reconstructed in a phase space of any dimension, but at present the topological tools can be applied only to attractors embedded in a threedimensional space. To compensate for this restriction, the topological tools reveal the mechanism responsible for producing chaotic behavior. In addition they are overdetermined, so that the results of an analysis can be rejected if well-defined self-consistency criteria are not met. Geometric and dynamical analysis methods do not reveal mechanism.

At the embedding stage, tests to determine whether a mapping is an embedding or not have been of two types in the past: geometric and dynamical. As for analyses, these tests for embeddings are applicable to any dimension. They all lack rejection criteria. We have recently proposed a topological test for embeddings [1]. This topological test is applicable only to three-dimensional mappings of data. However, this test supports strong rejection criteria.

There have been many claims of successful embeddings of data based on geometric measures (fractal dimensions, false near neighbors) or dynamical measures (Lyapunov exponents and dimensions, predictability), but few independent tests of whether such claims are valid. For this reason, in the present work we will compare the results of tests for embeddings using the three different tools currently available in three dimensions. We find that tests for embeddings based on geometric measures and dynamical measures fail to distinguish between mappings that are embeddings and those that are not. We discuss sources for such failures and the impact of noise on these tests. In view of our results in three dimensions where the topological test is available to test claims based on geometric and dynamical methods, we suggest that all claims for successful embeddings into three or higher dimensions, based on geometric or dynamical methods, be treated with the greatest skepticism.

This work is organized as follows. In Sec. II we review the tests for embeddings that have been proposed. In Sec. III we describe the two sets of data used for this comparison. In Secs. IV-VI we present the results of the geometric, dynamical, and topological tests for the mappings of these data sets. The real measures involved in the classical tests undergo intrinsic fluctuations from one data set to another. The statistics of these fluctuations are discussed in Sec. VII. In Sec. VIII we describe why the classical tests, depending on real measures averaged over the mapped attractor, can be insensitive to whether the mapping is or is not an embedding. Each test depends on values of one or more parameters that must be set before the test is implemented. Test results could depend on these parameter values. The parameters intrinsic to each type of test are discussed in Sec. IX. We compare the relative computational load for these three types of test in Sec. X. The effects of noise on the robustness of these tests is discussed in Sec. XI. In Sec. XII we summarize our findings: all assertions that a mapping is an embedding based on geometric or dynamical tests should be treated as provisional and regarded with skepticism until independent tests either reject or fail to reject such claims.

\section{REVIEW OF EMBEDDING TESTS}

The first step in the analysis of chaotic data is a search for a suitable embedding [2-4]. An embedding is a mapping of the data into a phase space $\mathbb{R}^{D}$ without self-intersections, so that each point in the attractor has a unique future and determinism is preserved [5]. An embedding creates a diffeomorphism between the original attractor and the reconstructed attractor. If the data are chaotic, $D \geqslant 3[6]$.

Mappings of scalar time series into $\mathbb{R}^{D}$ take the form $m(t) \rightarrow\left(x_{1}(t), x_{2}(t), x_{3}(t), \ldots, x_{D}(t)\right)$, where the coordinates $x_{i}(t)$ are functions of the observables. A number of mappings have been proposed. The default is the time delay mapping, in which $x_{i}(t)=m[t-(i-1) \tau]$, where $\tau$ is the time delay [2-4]. Another very useful mapping is the differential mapping, in which $x_{i}(t)=d^{(i-1)} m(t) / d t^{(i-1)}$ [7]. Other useful mappings take the form of mixtures of these two types-for 
example, $m(t) \rightarrow(m(t), d m(t) / d t, m(t-\tau))$, singular value decomposition (SVD) mappings, and Hilbert transform pairs. Classes of mappings have been reviewed in [8-12]. Projections of various types, involving Fourier or SVD decompositions, are particularly useful for vector time series. Projection of data from higher into lower dimensions [13] is similar in spirit to reductions of partial differential equations to sets of ordinary differential equations $[14,15]$.

Low-dimensional differential embeddings, when they can be found, offer a strong advantage over other types of embeddings. The advantage comes in the next natural step in the analysis. This is the construction of a model, a set of ordinary differential equations, that simulates the process generating the data. When a differential embedding is available, the derivative of each variable is the next variable $\left(d x_{i} / d t=x_{i+1}\right)$, so it is only necessary to model the dynamics of the last variable: $d x_{n} / d t=f\left(x_{1}, x_{2}, \ldots, x_{n}\right)$. For a time delay embedding it is necessary to construct $n$ functions, each of $n$ variables: $d x_{i} / d t=f_{i}\left(x_{1}, x_{2}, \ldots, x_{n}\right), i=1,2, \ldots, n$.

Once observed data have been mapped into a $D$-dimensional phase space it is necessary to determine whether the mapping is an embedding. A number of embedding tests have been used extensively in the past. They all share a common feature: they are implemented as a function of increasing dimension, a real statistic is averaged over the image of the attractor, and a mapping is declared an embedding when a threshold is reached. These classical tests are of two broad types: geometric and dynamical. Each type has many variants. The earliest test was geometric. It depended on computing geometric invariants [16] such as fractal dimensions and looking for "saturation of fractal dimensions" as a function of increasing dimension [17-19]. In principle, fractal dimensions are invariants under diffeomorphisms, so that they saturate at constant values with increasing dimension. In practice, this test was difficult to implement. As early as 1990, Ruelle cautioned about undue reliance on these unreliable measures [20]. Then in 1992 Lefranc and colleagues showed that correlation dimension estimates of experimental data were in practice not invariant under a simple logarithmic transformation [21].

Embedding tests depending on fractal dimension estimates were largely superseded in practice by the "false near neighbor" test [22]. This test looks at two points near each other in some mapping and determines whether they remain near each other as the dimension increases. If "yes," the two points are assumed near each other in the original attractor that generated the data. If "no," the two points are considered false near neighbors. A mapping is declared an embedding when a sufficiently small percentage of point pairs are determined to be false near neighbors.

Dynamical tests are similar in spirit. If a mapping is an embedding, then a point in $\mathbb{R}^{D}$ has a unique future. Predictability of the future from the present was implemented in the "bad prediction" test [23]. This same property was implemented in another way as a "test for determinism" [24]. In this test the phase space was decimated in each dimension and the uniqueness of the flow direction in each $D$-dimensional cube was estimated by computing inner products of tangent vectors to all trajectories passing through each cube. Both these dynamical tests declare a mapping to be an embedding when the relevant statistic reaches an appropriate value. Data requirements for the test for determinism grow exponentially with dimension $D$ and decimation degree. To average $n$ points per box in a $D$-dimensional mapping with decimation degree $d, n d^{D}$ points are required.

In part because of large data requirements, this class of tests was largely supplanted by tests depending on Lyapunov exponent estimates [5,25-29] and Lyapunov dimension estimates [30] that are derived from them. The basic idea is that as the dimension increases, estimates of the largest Lyapunov exponent become reliable when and after an embedding is achieved. A major problem is that the number of Lyapunov exponents is equal to the dimension of the mapping. Above the correct embedding dimension there are spurious exponents, and these can be larger than the largest Lyapunov exponent $[31,32]$. We concentrate our study on Lyapunov exponent tests with the understanding that other dynamical tests for embeddings are in principle still possible.

The topological test is applicable to families of three dimensional mappings. The essential idea is that for the mapping to be an embedding, each point must have a unique future: self-intersections are forbidden. If the chaotic attractor undergoes self-intersections as some parameter in the family varies, then the unstable periodic orbits that exist in abundance in the attractor will also undergo intersections. When two unstable periodic orbits undergo a selfintersection, the minimum distance between these orbits goes to zero. In such cases, a plot of the minimum distance between two orbits as a function of the mapping parameter shows a dip towards zero, followed by a rise from zero after the crossing. We call such events "zero crossings." The zero crossing may be resolution limited. In such cases, the zero crossing can be confirmed by computing an important topological index. The Gauss linking number changes by \pm 1 at a zero crossing, or at an intersection of two unstable periodic orbits $[7,11,12,33]$.

The basic idea of and algorithm for the new topological test for embedding is simple to state [1]. (i) Search through the time series for unstable periodic orbits. This can be done by the method of close returns before any embedding is attempted $[11,12,34]$. (ii) Choose a family of mappings depending on some parameter and map these orbits into $\mathbb{R}^{3}$. (iii) Compute the minimum distance between each pair of mapped orbits in the parameter range. The mapping cannot be an embedding where any of these minima show zero crossings. If a zero crossing is uncertain (cf, Fig. 12 below), compute the linking number $[11,12,33]$ of the two orbits on each side of the apparent crossing. At a real crossing the linking number changes by \pm 1 . In parameter regions where the linking numbers of two or more orbit pairs change frequently - that is, where many zero crossings occur-we can reject the hypothesis that the mapping is an embedding. Where there are no zero crossings, the linking numbers remain unchanged and it is not possible to reject the hypothesis that the mapping is not an embedding (in friendlier words, the mapping could be an embedding).

Confirming that the mapping is an embedding follows from a topological analysis of the mapped attractor. This type of analysis can even be applied to a single mapping not belonging to a family of mappings, to determine whether it is 
an embedding or not. Details are available in [7,11,12].

Behind all these tests lurk the embedding theorems. These guarantee that if the dynamics is produced by an $n$-dimensional dynamical system, the data describing the dynamics can always be embedded in $\mathrm{R}^{D}$ for $D$ sufficiently large. These theorems are based on the idea that if the dimension is sufficiently large, there is enough room in phase space so that self-intersections typically ("generically") do not occur. The simplest estimate $D \geqslant 2 n+1$, based on genericity, was reduced to $D \geqslant 2 n$ by Whitney [35] and reduced once again to $D>2 d_{A}$ [36], where $d_{A}$ is an appropriate fractal dimension. For the Lorenz attractor [15] with $d_{A}=2.06$ for $(R, \sigma, b)=(28.0,10,8 / 3)$, these theorems guarantee that an embedding into $\mathbb{R}^{D}$ with $D \geqslant 5$ can always be found. As pointed out by Abarbanel and collaborators [8,9,22], for those interested in knowing the minimum (necessary) dimension for having an embedding, to have a sufficient condition is useless. If the dynamics is three dimensional, we need an algorithm for constructing a three-dimensional embedding rather than a theorem stating that a five-dimensional embedding exists.

Once a suitable embedding has been determined, the embedded data can be analyzed. The analysis procedures are of three broad types: geometric [18], dynamical [25], and topological [7]. Geometrical analyses focus on computing the spectra of fractal dimensions or the fraction of false near neighbors. Dynamical analyses focus on estimating Lyapunov exponents and predictability indices. These calculations can be carried out for any value of the dimension $D$. The results are real numbers with no underlying statistical theory to provide believable error estimates (error bars are "educated guesses" [19]) and no information about the mechanism that generates chaotic behavior. Topological analyses can be carried out only when $D=3$. The results are overdetermined and therefore contain their own rejection criteria $[7,11]$, an assertion that cannot be made for the other two analysis methods. Further, these analyses reveal the nature of the bending and folding mechanism [37] or the stretching and squeezing mechanism [38] responsible for generating chaotic behavior.

\section{COMPARISON DATA SETS}

We use two data sets to compare the three types of tests for embedding. In neither case do we employ a standard time delay embedding. For this reason the standard types of analyses employed with geometric and dynamical testssaturation of some real measure-are not available.

Data taken during a fluid experiment in a BenardMarangoni configuration [39] were analyzed [40] and modeled as a periodically driven Takens-Bogdanov oscillator [41] in the following form:

$$
\begin{gathered}
\dot{x}=y, \\
\dot{y}=(\mu x+\nu y) f(\phi)-x^{3}+x^{2} y,
\end{gathered}
$$

$$
\dot{\phi}=\omega
$$

where $f(\phi)=1+\epsilon \cos (\phi)$. The parameter values were taken as $(\mu, \nu, \epsilon, \omega)=(1.0434,-1.0,0.45,0.399)$. A single scalar time series $x(t)$ was produced by this model and mapped from $\mathbb{R}^{1}$ into $\mathbb{R}^{3}$ a with mixed differential-delay type mapping that was explicitly $x(t) \in R^{1} \rightarrow(x(t), d x(t) / d t, x(t-\tau))$ $\in \mathbb{R}^{3}[41]$. The mapping parameter is the single time delay $\tau$. For some values of the delay the mapping is an embedding while for others it is not. The analysis leading to these conclusions has been described in [42] and will be reviewed in Sec. VI.

A three-dimensional differential embedding $x(t)$ $\rightarrow(x, \dot{x}, \ddot{x})$ would have been preferable for reasons given above, but was not used because of noise involved in computing $\ddot{x}[7,39]$. A standard time delay embedding $x_{i}(t)=x[t$ $-(i-1) \tau]$ would have involved determination of three forcing functions $d x_{i} / d t=f_{i}\left(x_{1}, x_{2}, x_{3}\right)$. For this reason the mixed differential-delay embedding was adopted [41].

Previous study $[40,42]$ has shown that the one-parameter family of mappings depending on the single time delay $\tau$ is an embedding for some values of $\tau$ and not for others. We show below that the topological test can distinguish between values of $\tau$ for which the mapping is an embedding and for which it is not, while the geometric and dynamical tests fail to make this distinction.

The second data set that we used to compare the three methods of testing for embedding is a four-vector time series produced by the dynamical system [43]:

$$
\begin{gathered}
\dot{X}=\sigma(-X+Y)-7.1111 \beta U, \\
\dot{Y}=(R / \nu) X-Y-X Z, \\
\dot{Z}=-\nu Z+X Y, \\
\dot{U}=-\Lambda U+X .
\end{gathered}
$$

This system is a modification of the Malkus-Robbins dynamo equations [involving only $(X, Y, Z)]$ which were originally introduced to model the action of a self-exciting dynamo [44-46]. The extension is to include the variable $U$, which represents the angular speed of the motor of the dynamo [45]. For $\beta=0$ there is no feedback from the $U$ subsystem into the $(X, Y, Z)$ subsystem, which behaves like a Lorenz attractor. The dynamics was studied for $(\sigma, \nu, R)$ $=(10,8 / 3,74.667)$, where the Lorenz equations generate a chaotic attractor and $\Lambda=3$.2. In the range $0 \leqslant \beta<7.9$, Eq. (2) generates a chaotic attractor with Lyapunov dimension $d_{L}$ $\simeq 2.2$ [43]. A boundary crisis destroys the attractor at $\beta$ $\simeq 7.9$.

In this case all four time series $(X(t), Y(t), Z(t), U(t))$ are available. This is not usual for experimental systems. The question that drives this analysis is one of reducing complexity. Is it possible to simplify the study of this dynamical system by projecting the attractors into a lower- (three-) dimensional space? In this case we do not have a single attractor and a family of mappings into $\mathrm{R}^{3}$. Rather, there is a one-parameter $(\beta)$ family of attractors, and we seek to deter- 
mine whether the projection into some $\mathbb{R}^{3}$ subspace is an embedding for all, some, or no values of the parameter $\beta$ [43].

We used topological tests for embeddings and topological analyses to determine under what conditions the two projection mappings $(X, Y, Z, U) \rightarrow(X, Y, Z)$ and $(X, Y, Z, U)$ $\rightarrow(X, Y, U)$ were or were not embeddings. We found the first projection failed to be an embedding in the range $0.6<\beta$ $<5$.4. In this parameter range the projected attractor undergoes self-intersections (cf. Fig. 15 below) and the unstable periodic orbits in the attractor undergo many zero crossings. The second projection $(X, Y, Z, U) \rightarrow(X, Y, U)$ was an embedding for all values of the parameter $\beta$. The attractor undergoes no self-intersections as a function of $\beta$ in this projection. The analysis leading to these conclusions has been described in [43] will be reviewed in Sec. VI.

The two sets of time series studied below have been produced by Eqs. (1) and (2).

\section{GEOMETRIC TESTS}

Two types of geometric tests were carried out. One depends on estimating the fraction of false near neighbors as a function of the appropriate parameter. The other involves estimating the correlation dimension, also as a function of the mapping parameter.

\section{A. False near neighbors}

The false nearest neighbors (FNN) test was applied following the usual procedure [22]. A point $\mathbf{x}(i)$ is chosen, and all points $\mathbf{x}(j)$ are searched over whose first $k$ coordinates are within a distance $\boldsymbol{\epsilon}$ of each other: $\left|x_{r}(i)-x_{r}(j)\right|<\epsilon, r$ $=1,2, \ldots, k$. For each such $\mathbf{x}(j)$ the $k+1$ st coordinates of $\mathbf{x}(i)$ and $\mathbf{x}(j)$ are compared and the points at $i$ and $j$ are declared true neighbors if $\left|x_{k+1}(i)-x_{k+1}(j)\right|<5 \epsilon$ and false neighbors otherwise. The number of true and false neighbors is computed for each point $\mathbf{x}(i)$ and the ratio of false neighbors to the total number of its neighbors is computed. As $k$ increases, this ratio decreases, and when it gets "small enough," an embedding is declared. In this estimate $\epsilon$ was chosen as $1 \%$ of the diameter of the attractor. We point out that $\epsilon$ and 5 are reasonable but arbitrary input parameters.

\section{Fluid model}

In order to carry out the FNN test on the fluid data to see if a three-dimensional embedding exists we need at least four coordinates. The first three were prescribed by the family of mappings chosen: $(x(t), d x(t) / d t, x(t-\tau))$. As a fourth coordinate we chose $x_{4}(t)=x(t-2 \tau)$ in the spirit of standard time delay embeddings. To check that the codes developed to perform this computation were robust, we included a fifth coordinate, chosen again in the spirit of standard time delays as $x_{5}(t)=x(t-3 \tau)$. If the first three coordinates did not provide an embedding, the first four should, so that the fraction of FNN in going from four to five dimensions should fall to approximately zero. This provided a test of the computational procedure. The test was carried out for data sets of

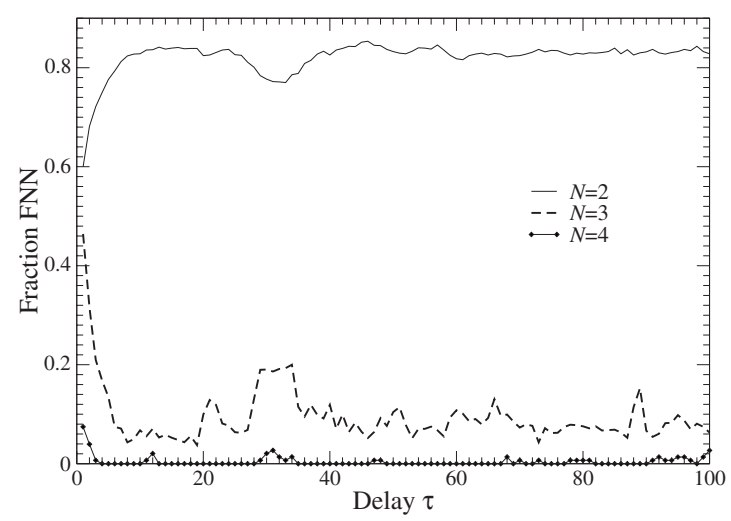

FIG. 1. Fraction of false near neighbors as a function of time delay $\tau$ for embedding dimensions $N=2,3,4$.

length 5000 points. Some larger data sets were used without the results being altered in any way.

We computed the fraction of FNN in going from $N=2$ to 3 dimensions, $N=3$ to 4 , and $N=4$ to 5 dimensions. The results are shown in Fig. 1, plotted as a function of the delay parameter $\tau$. For $N=2$ the fraction of false near neighbors is in excess of 0.5. This is to be expected, as two-dimensional systems cannot behave chaotically. For $N=4$ the fraction of FNN is zero or almost zero for all delays. This is also expected, as the system is three dimensional [42]. For $N=3$ there is a substantial fraction of FNN. Further, this fraction does not show any clear signatures that a three-dimensional embedding occurs for some values of the delay $\tau$ in this region and not for others. This curve is a clear indication that the FNN test fails to distinguish between mappings that are embeddings and those that are not in the present case.

\section{Dynamo model}

The FNN test was also applied to the dynamo data $\mathbf{x}(i)$. One data set was chosen in the form $(X, Y, Z, U)$. As applied, we tested whether two points with indices $i$ and $j$ whose $(X, Y, Z)$ coordinates were close in the $\mathrm{R}^{3}$ subspace were also close in the full space $\mathbb{R}^{4}$. The FNN test was carried out as a function of the parameter $\beta$. The FNN test was also carried out on the reordered data $(X, Y, U, Z)$ to test in the same way whether the projection into the $(X, Y, U)$ subspace was an embedding.

Results of this test are shown in Fig. 2. This figure shows the FNN ratio as a function of the parameter $\beta$. The detailed nature of these curves depends on the choice of input parameters $\epsilon$ and 5, but the conclusions drawn do not. The curve for the $(X, Y, Z)$ projection shows nothing significant at either $\beta=0.6$ or $\beta=5.4$, where the transitions occur from the projection being an embedding to not being an embedding [1]. In the range $0.6<\beta<5.4$ the projected attractor exhibits self intersections.

If the hypothesis is formulated-this projection is an embedding-it is not unreasonable to reject this hypothesis when the variance around the mean stops hitting the $\beta$ axis. For the $(X, Y, Z)$ projection this occurs with $\beta$ decreasing at $\beta \simeq 3.2$. One would reject this hypothesis for $\beta<3.0$ and fail to reject it for $\beta>4.0$. Both conclusions are incorrect. 


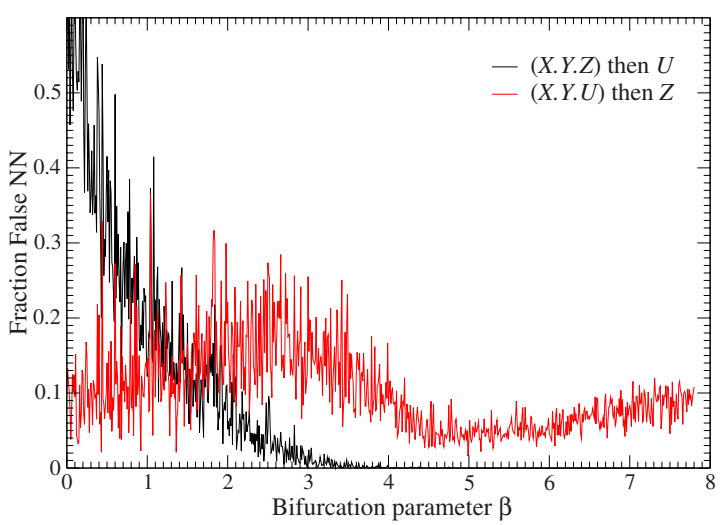

FIG. 2. (Color online) Fraction of false near neighbors under the projections to the $(X, Y, Z)$ (dark) and $(X, Y, U)$ (light) subspaces.

For the $(X, Y, U)$ projection, the FNN ratio variations about the mean never include zero. Once again, if the hypothesis is formulated-this projection is an embeddingthe hypothesis would be rejected for all values of $\beta$. It is known from topological analyses that the projection $(X, Y, Z, U) \rightarrow(X, Y, U)$ is an embedding for all values of $\beta$ [43].

The results are that the FNN test failed to determine conditions under which these projections are embeddings.

\section{B. Fractal dimension}

Fractal dimension tests are usually carried out on time delay embeddings, increasing the dimension of the embedding until changes in the form of the correlation integral curve become small enough. We cannot adopt this strategy here, since we seek to learn when a three dimensional mapping is an embedding. As a result, the only quantitative criterion available to us is to make a judgement based on the value of the computed fractal dimension. We understand that using fractal dimension as an indicator that an embedding has been achieved is often quite different from the actual computation of the fractal dimension. Unfortunately, the possibility is not open to us to raise dimension until changes in the correlation integral curve become small enough, so we can only base our hypothesis tests on the estimated value of this statistic in the mapping into $\mathbb{R}^{3}$ : the fractal dimension $D_{2}$.

The correlation integral $D_{2}$ was computed following standard recipes $[17,18]$. All such calculations were done simultaneously with estimates of the largest Lyapunov exponent, as explained in Sec. V below. A plot of the logarithm of the correlation integral $\sum_{i, j} \Theta(\epsilon-|\mathbf{x}(i)-\mathbf{x}(j)|)$ versus the logarithm of the size $\epsilon$ is shown in Fig. 3. All logarithms in this work are computed to base $e$. The correlation dimension is the slope of the linear part of this plot. All plots of this type obtained using the algorithm suggested by Rosenstein et al. [29] had a very-well-defined linear region from which it was easily possible to obtain a good estimate of $D_{2}$. This integral was computed for the projection of the attractor at $\beta=4.0$ from the full $(X, Y, Z, U)$ space into the $(X, Y, U)$ subspace. All such integrals showed a well-defined linear region, which was used to estimate $D_{2}$.

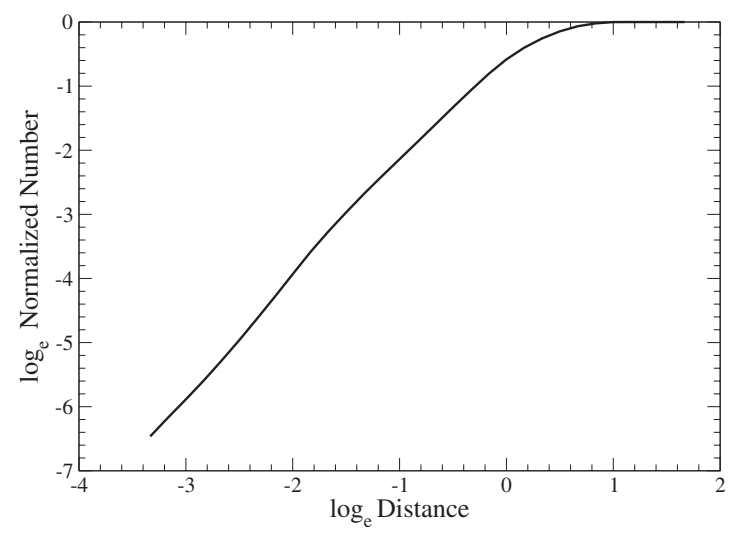

FIG. 3. Logarithm of the correlation integral as a function of the logarithm of the distance $\epsilon$ for the three-dimensional projection $(X, Y, Z, U) \rightarrow(X, Y, U)$ of the dynamo model for $\beta=4.0$.

\section{Fluid model}

Estimates of the correlation dimension for a threedimensional differential-delay vector obtained from the scalar fluid data are shown in Fig. 4 as a function of the time delay $\tau$. For $\tau=0$ the mapping is not an embedding and the $D_{2}$ estimate falls below the threshold of 2.0 [30]. For $\tau \geqslant 2$ this estimate is larger than 2.0. Further, the curve shows no particularly strong features to suggest that for certain values of the delay $\tau$ the mapping is an embedding while for others it is not. If we were to formulate the hypothesis test-this mapping is an embedding-we would reject this hypothesis for values $D_{2}<2$.0. On the basis of the results in Fig. 4 we would fail to reject the hypothesis for $2<\tau<100$. This conclusion will be shown to be incorrect below.

\section{Dynamo model}

The correlation dimensions for the two projections $(X, Y, Z, U) \rightarrow(X, Y, Z)$ and $(X, Y, Z, U) \rightarrow(X, Y, U)$ were computed as a function of $\beta$. The results are plotted in Fig. 5 for the $(X, Y, Z)$ projection and in Fig. 6 for the $(X, Y, U)$ projection. The variance observed in these plots is not a function of changing $\beta$; it is intrinsic, a function of changing the data set. This is discussed further in Sec. VII. In both

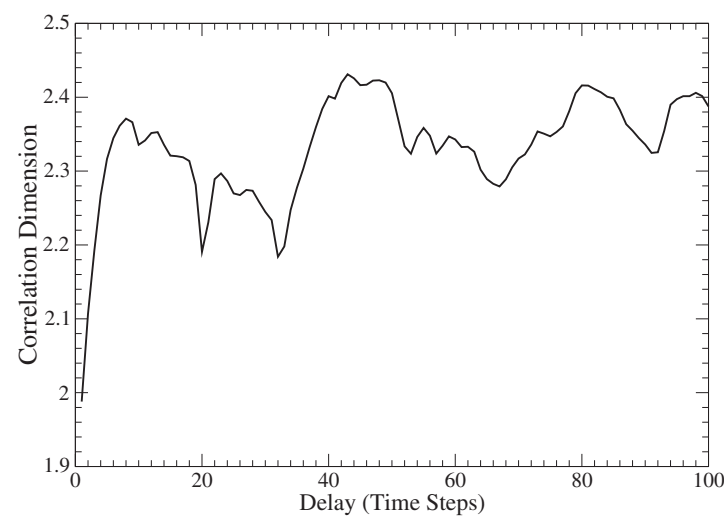

FIG. 4. Correlation dimension estimate, as a function of time delay, for a three-dimensional differential-delay mapping of $x(t)$ from the fluid model. 


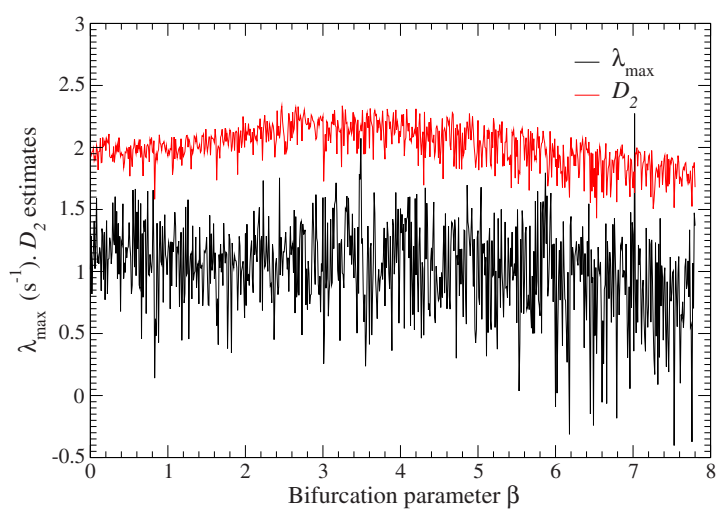

FIG. 5. (Color online) Estimates of the fractal dimension $D_{2}$ (top curve) and the LLE (bottom curve) for the $(X, Y, Z)$ projection of the dynamo model.

cases the correlation dimension curve $D_{2}$ shows less variance than the largest Lyapunov exponent curve.

There is no special signal in the $D_{2}$ curve for the $(X, Y, Z)$ projection that the mapping is not an embedding in the range $0.6<\beta<5.4$.

The hypothesis test-the projection is an embeddingcan be formulated and treated as described above. In the $(X, Y, Z)$ case we reject the hypothesis that there is an embedding where there actually is one ( small $\beta$, large $\beta$ ) and fail to reject this hypothesis when the projection is not an embedding. In the $(X, Y, U)$ case $D_{2}$ is always less than 2.0, but the mapping is always an embedding. As a result, under this test completely incorrect conclusions are reached.

\section{DYNAMICAL TEST}

The dynamical test that was carried out involved estimating the largest Lyapunov exponent (LLE) as a function of the appropriate parameter. The LLE was estimated using an algorithm proposed by Sato et al. [26] and implemented using suggestions made by Rosenstein et al. [29]. A small modification in this algorithm, also proposed in [29], was used to estimate the correlation dimensions that are presented above.

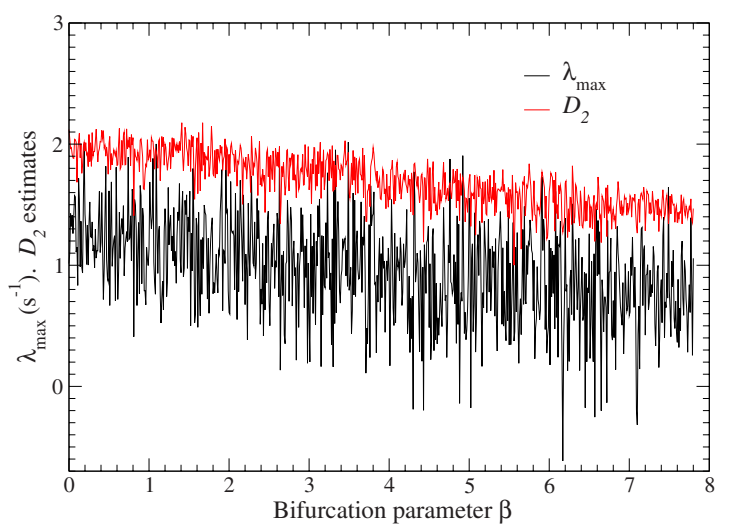

FIG. 6. (Color online) Estimates of the fractal dimension $D_{2}$ (top curve) and the LLE (bottom curve) for the $(X, Y, U)$ projection of the dynamo model.

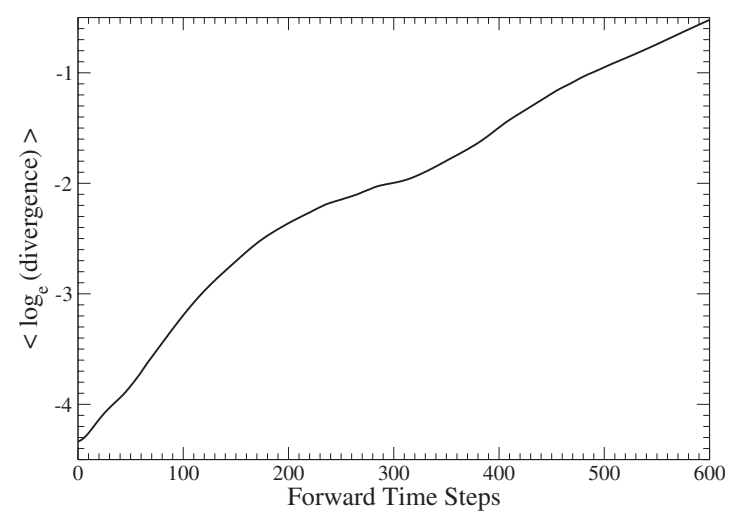

FIG. 7. Estimate of the logarithm of the divergence as a function of the number of forward time steps for the projection $(X, Y, Z, U) \rightarrow(X, Y, U)$ of the dynamo model at $\beta=4.0$. The largest Lyapunov exponent is to be estimated from the slope of the "locally linear" region. This curve was computed simultaneously with the curve shown in Fig. 3, which has a single linear region.

Briefly, we look at discretely sampled time series in three variables $\left(x_{1}, x_{2}, x_{3}\right)_{i}$, fix $i$, and search over the entire time series for the nearest neighbor $j$. Once found, we compute the distance between these two points as they propagate $k$ steps into the future ( $k$ up to 2000 steps). The logarithm of the distance is computed for each $k$. These measures are averaged over the entire data set, $i$. The "linear portion" of the $\left\langle\log _{e}\right.$ (divergence) $\rangle$ vs $k$ curve is fitted with a straight line, and the slope of this fit, divided by $d t$, is an estimate of the largest Lyapunov exponent. Our implementation of this algorithm was verified by reproducing Fig. 2 in [29]. That curve has more than one "linear portion," and each such portion is far from linear. A straight line of the form $y=a+b x$ was fitted to various parts of that curve using a moving average window and sliding center. By choosing an appropriate part of the curve it was possible to reproduce their estimate of 1.50 for the largest Lyapunov exponent of the Lorenz attractor at particular parameter values using windows of various halfwidths from 5 to 100 . However, other parts of the curve and other window widths provided estimates ranging from about 3 down to almost zero.

Plots of $\left\langle\log _{e}\right.$ (divergence) $\rangle$ vs $k$ for three-dimensional projections of the dynamo data always had two, sometimes three, and occasionally four "linear portion" regions, and each region usually had small fluctuations. One such curve is shown in Fig. 7. This curve was computed simultaneously with the curve shown in Fig. 3. These results are typical of the dynamo data: the correlation integral curves usually showed a single linear region while the $\left\langle\log _{e}\right.$ (divergence) $\rangle$ curves had several.

The corresponding $\left\langle\log _{e}(\right.$ divergence $\left.)\right\rangle$ curves for the fluid data were remarkably different. One such curve is shown in Fig. 8. This curve has no locally linear regions under the most optimistic assumptions. Estimates of the $\left\langle\log _{e}(\right.$ divergence $\left.)\right\rangle$ curves were made by smoothing over long intervals. We do not regard such estimates as very significant.

We do not know of any hypothesis test on whether a mapping is an embedding that is reasonable to formulate 


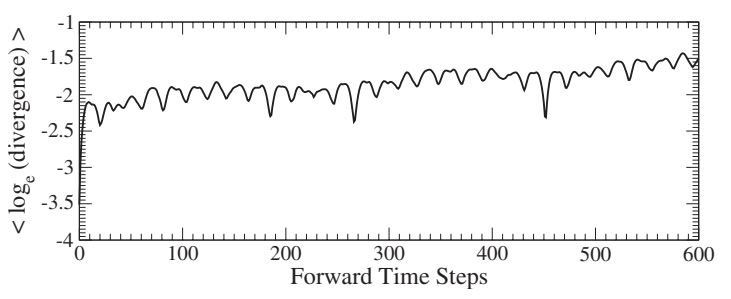

FIG. 8. Estimate of the logarithm of the divergence as a function of the number of forward time steps for the fluid data. The largest Lyapunov exponent is to be estimated from the slope of the "locally linear" region.

using the largest Lyapunov exponent as a quantitative measure.

\section{A. Fluid model}

We estimated $\left\langle\log _{e}\right.$ (divergence) $\left.)\right\rangle$ as a function of the number of forward time steps for time delays $1 \leqslant \tau \leqslant 100$. Each of these curves resembled that shown in Fig. 8. We felt it was meaningless to try to estimate the LLE from such data. Nevertheless, we fixed a range of forward time steps $(300 \leqslant k$ $\leqslant 500$ ) and used this range to fit a straight line $y=a+b x$ and extract the slope $m$. The result is shown in Fig. 9. There are no clear signatures in this curve that the mapping is an embedding for certain values of the delay and not for others.

\section{B. Dynamo model}

We computed the largest Lyapunov exponents for both the $(X, Y, Z)$ and $(X, Y, U)$ projections for all values of $\beta$ in the interval $[0,7.8]$ with $\Delta \beta=0.01$. An interval was chosen (different for the two embeddings) where all plots seemed to have a linear region. The results are shown in Figs. 5 and 6. In both cases the LLE is the lower curve with the larger variance. Neither curve shows any remarkable behavior that would distinguish between a projection that is an embedding and one that is not.

\section{TOPOLOGICAL TESTS}

The topological test depends on finding and using the unstable periodic orbits in the chaotic data set. Such orbits can be identified in scalar time series even before an embedding into $\mathbb{R}^{3}$ is attempted $[7,11,12]$. The topological test for embedding looks for stability of the linking numbers between

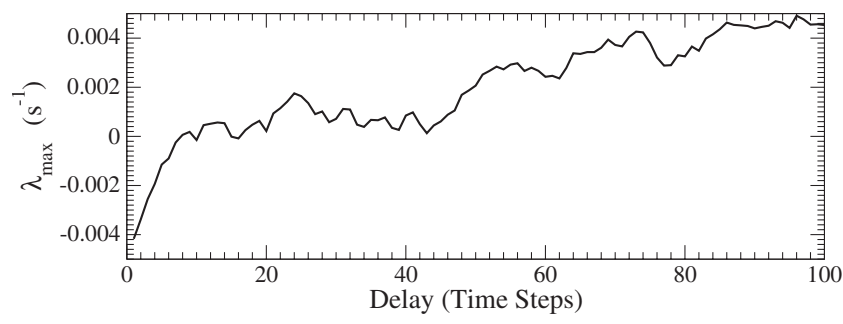

FIG. 9. Estimate of the LLE for differential-delay mapping of the scalar time series $x(t)$ from the fluid model as a function of delay $\tau$.

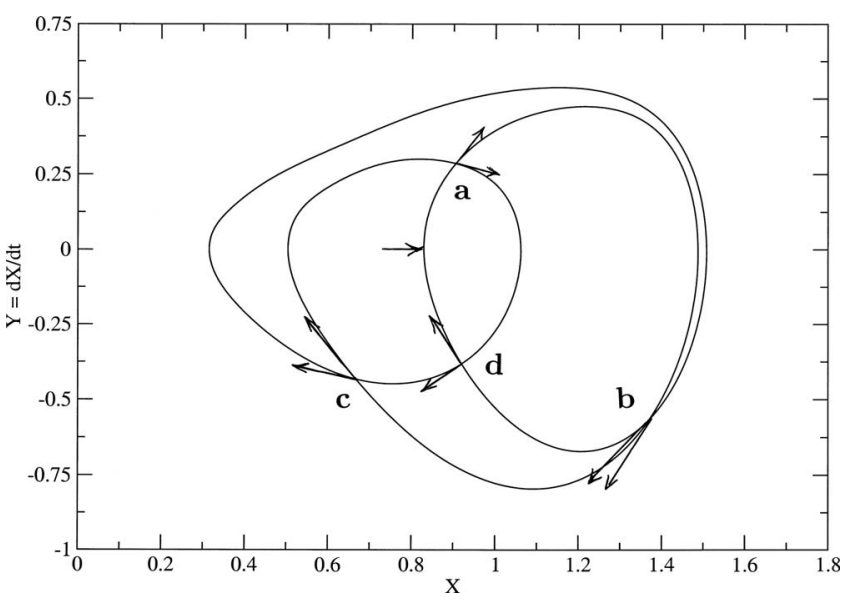

FIG. 10. Projection of the lowest period orbit onto the $x-\dot{x}$ plane is independent of the time delay $\tau$ in the mixed differential-delay mapping. There are four crossing points in this projection.

all pairs of unstable periodic orbits. Even more simply, it looks for intersections of such orbits or even intersections of an orbit with itself. Intersection of two orbits is identified by computing the minimum Euclidean distance between two orbits and plotting this distance as a function of the mapping parameter. Intersections appear as a V-shaped almost intersection of the minimum distance curve with the mapping parameter axis. In regions where zero crossings occur and linking numbers of unstable periodic orbits change, the mapping cannot be an embedding.

\section{A. Fluid model}

The topological test for embedding of data from the fluid model was simplified as follows. The lowest-period orbit in the attractor was located before any embedding was made. Its projection into the $x-\dot{x}$ plane is independent of $\tau$ and is shown in Fig. 10. This orbit is "in the attractor," so is surrounded by a tube (a torus) that contains the attractor. If this orbit undergoes a self-intersection for $\tau=\tau_{1}$, the tube, and the attractor it contains, undergo self-intersections in an interval containing $\tau_{1}$. The mapping cannot be an embedding in this interval of parameter values.

The projection of this curve onto the $x_{1}-x_{2}$ plane is shown in Fig. 10. There are four crossing points in this projection. At each crossing point the first two coordinates of each of the two segments that cross are equal. If the third coordinates are also equal, the curve has a self-intersection at that point.

The difference between the third coordinates of the two segments is shown in Fig. 11. The difference in the $x_{3}$ coordinates at each of the four crossing points $a-d$ in the $x-\dot{x}$ projection is shown in Fig. 11 as a function of $\tau$ $=T_{1}(j / 1024)$, where $T_{1}$ is the period of this orbit. When this distance is zero the orbit has a self-intersection and the mapping cannot be an embedding. We have computed topological invariants for periodic orbits in this attractor and their changes from one embedding interval to another. In each instance the change in global torsion [11,12] is $\Delta G T= \pm 2$. This is consistent with a change in the extrinsic embedding 


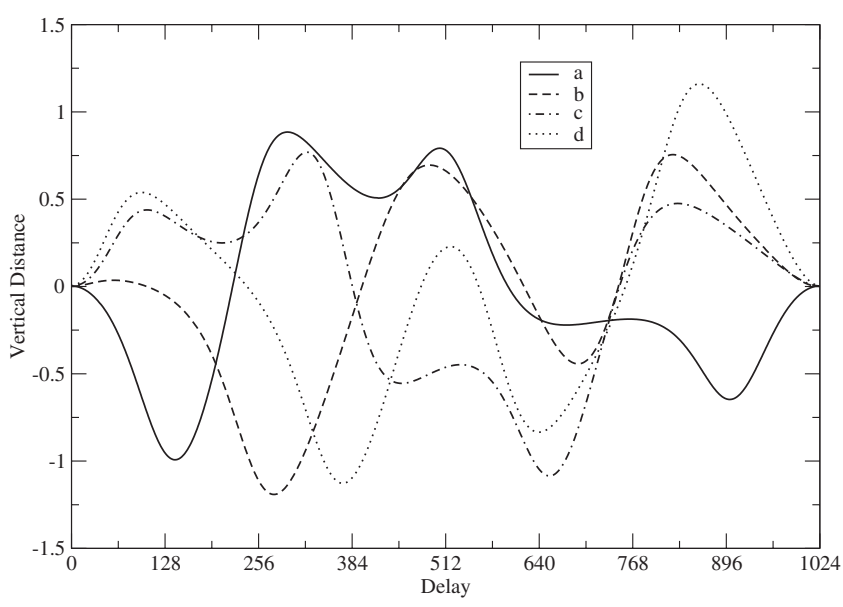

FIG. 11. The difference between the coordinates $x_{3}$ of the two segments that cross, at each of the four crossing points, is shown, plotted as a function of the time delay $\tau$. When this difference is zero the orbit has a self-intersection and the mapping fails to be an embedding in a region around the intersection point.

caused by a self-intersection of the chaotic attractor (cf, Ref. [42], Fig. 9).

\section{B. Dynamo model}

We used topological tests to determine whether the projections of the attractor generated by Eq. (2) from $\mathbb{R}^{4}$ into $\mathbb{R}^{3}$ given by $(X, Y, Z, U) \rightarrow(X, Y, Z)$ and $(X, Y, Z, U)$ $\rightarrow(X, Y, U)$ are in fact embeddings. We did this by extracting periodic orbits from the chaotic time series in $\mathbb{R}^{4}$ by the method of close returns $[34,47]$. A set of these orbits was followed adiabatically as the parameter $\beta$ varied through the range $0 \leqslant \beta<7.9$. The minimum distance and the linking numbers between all pairs of orbits were determined as a function of $\beta$ for each of the two projections.

In Fig. 12 we show the minimum distances between two unstable periodic orbits over a limited range of $\beta$ values, $0.25 \leqslant \beta \leqslant 1.5$. These three minimum distances were computed in the full space $(X, Y, Z, U)$ and in the two three-

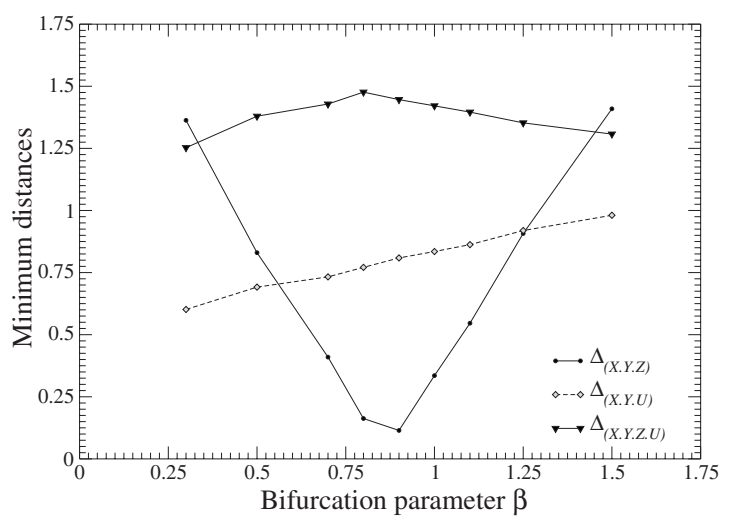

FIG. 12. Minimum distance between two orbits as a function of $\beta$ in the chaotic attractor in $R^{4}$ and under two projections into three-dimensional spaces. Parameter values: $(R, \sigma, \nu, \Lambda)$ $=(74.667,10,8 / 3,3.2)$. dimensional subspaces $(X, Y, Z)$ and $(X, Y, U)$.

In the four-dimensional space the minimum distance is smooth, varying between 1.25 and 1.5. This is expected and consistent with a theorem that curves in spaces of dimension greater than 3 do not intersect [12].

In the three-dimensional space $(X, Y, U)$ the minimum distances are also bounded away from zero. In fact, in this projection the minimum distance between any pair of the orbits we followed was bounded away from zero for all values of $\beta$. This shows that all the periodic orbits we studied have unchanging topological organization for all values of $\beta$. This allows us to conclude that the chaotic attractor undergoes no self-intersections in this projection, so that this projection is compatible with an embedding.

The situation is quite different for the projection $(X, Y, Z, U) \rightarrow(X, Y, Z)$. The minimum distance function dives steeply to zero approaching $\beta \simeq 0.85$ and rapidly increases from zero above 0.85 . The zero crossing is elegantly estimated by reflecting either of these linear sections in the horizontal axis and connecting the dots. The failure to reach zero is a resolution problem. The confirmation of a zero crossing is made by computing the linking numbers of these two orbits on either side of the crossing. The linking numbers differ by one, so the crossing is real. These two orbits exhibit many other zero crossings in the range not shown in this figure $(1.5<\beta<5.4)$. Other orbits exhibit zero crossings in the range $0.6<\beta<5.4$. From this we conclude that in the range $0.6<\beta<5.4$ the strange attractor undergoes a selfintersection, forcing the orbits in it to exhibit many intersections. Outside this range there are no zero crossings, selfintersections no longer occur, and the projection is compatible with an embedding.

These results allow us to reject the hypothesis that the projection $(X, Y, Z, U) \rightarrow(X, Y, Z)$ is an embedding in the interval $0.6 \leqslant \beta \leqslant 5.4$. We cannot reject the hypothesis that the projection $(X, Y, Z, U) \rightarrow(X, Y, U)$ is an embedding throughout the entire range $0.0 \leqslant \beta \leqslant 7.9$. Neither can we reject the hypothesis that the projection $(X, Y, Z, U) \rightarrow(X, Y, Z)$ is an embedding outside the interval $0.6 \leqslant \beta \leqslant 5.4$.

Where we could not rule out the hypothesis that the mapping is an embedding, it was subsequently determined to be an embedding by carrying out a topological analysis $[11,12]$ on the periodic orbits and identifying a branched manifold which reproduced all linking numbers and correctly predicted additional linking numbers [43].

\section{STATISTICAL TESTS}

Fluctuations in the values of the estimates of the LLE and $D_{2}$ in Figs. 5 and 6 are not due to changing the value of $\beta$. They vary with the data set used. The LLE and $D_{2}$ were computed for data sets in the three variables $(X, Y, Z)$ of length 5000 with $\beta=4.0$. This was repeated 1001 times. The results are shown in Fig. 13. The variance around the $D_{2}$ estimate is tighter than around the LLE estimate. This possibly reflects the fact that curves used to determine $D_{2}$ always had one well-defined locally linear section, while those used for LLE estimates never did.

The mean and variance were computed for each of the two data sets shown in Fig. 13. Each data set was scaled in 


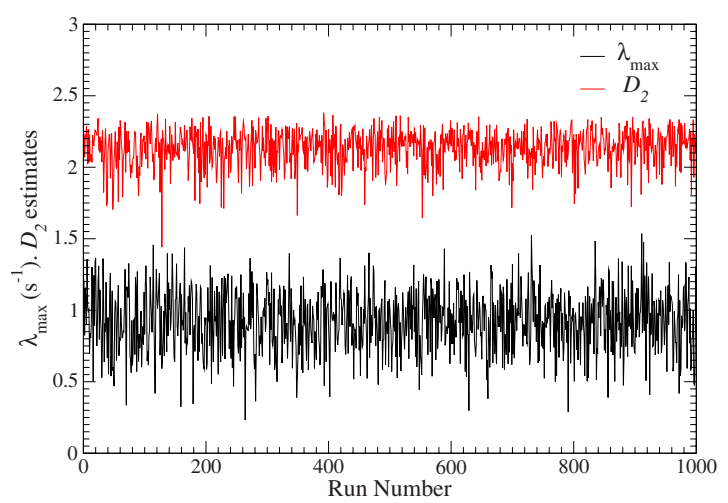

FIG. 13. (Color online) Estimates of LLE (bottom curve) and $D_{2}$ over 1001 projections into $(X, Y, Z)$ with $\beta$ fixed at 4.0.

the usual way, removing the mean and dividing the difference by the standard deviation. The results are binned in boxes of width 0.1 . The result is shown in Fig. 14. To test Gaussianity we did a $\chi^{2}$ test on these two distributions against a null hypothesis of normality. We found for the $D_{2}$ and LLE estimates values of 0.75 and 3.17 per degree of freedom. We fail to reject normally distributed fluctuations in the case of correlation dimension estimates and soundly reject it for LLE estimates.

\section{SENSITIVITY OF TESTS}

Figure 15 shows a simple "cardboard model" representation of the geometric structure created by the projection $(X, Y, Z, U) \rightarrow(X, Y, Z)$ for $\beta=3.8$ when it is not an embedding. The dark line indicates the region in the $(X, Y, Z)$ space where the two lobes of the attractor undergo selfintersection. This set has dimension not greater than 2 $\times 2.2-3 \simeq 1.4$ [48]. This estimate comes from genericity arguments. If two manifolds of dimension $p$ and $q$ are embedded in $\mathbb{R}^{N}$ and intersect, they intersect in a manifold of dimension $p+q-N \geqslant 0$. For this self-intersecting chaotic attractor in $\mathrm{R}^{4}, p=q \simeq 2.2$, and this is an upper bound on the

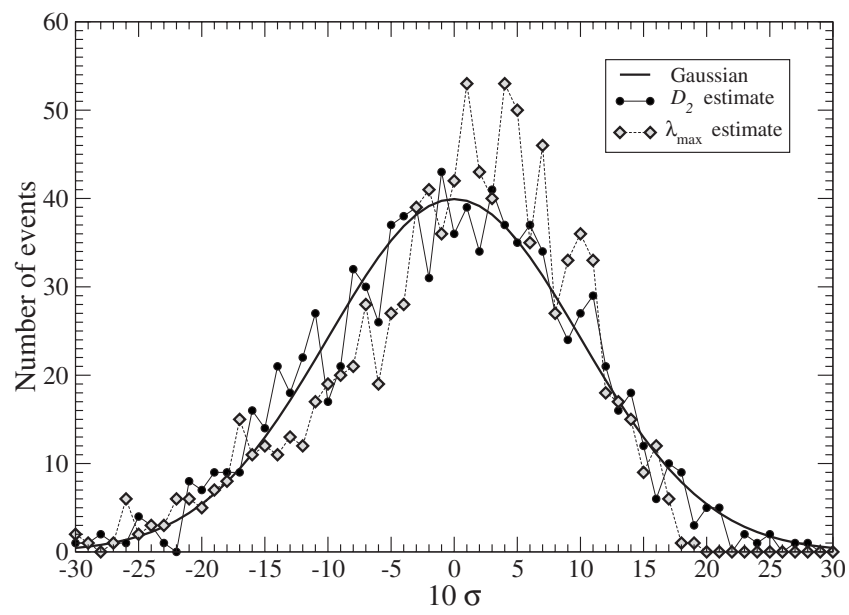

FIG. 14. Comparison of the suitably normalized statistical distribution of the LLE and $D_{2}$ estimates with the Gaussian normal distribution.

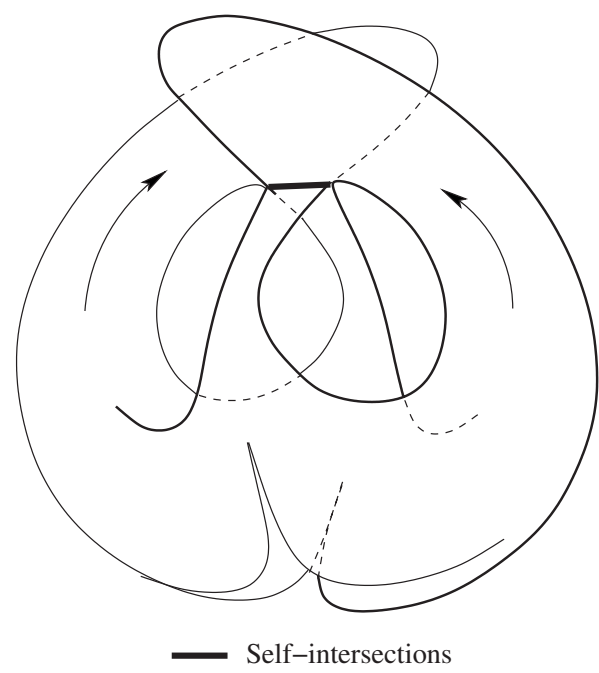

FIG. 15. Cardboard model of the self-intersection of the projection of the chaotic attractor into the $(X, Y, Z)$ subspace for $0.6<\beta$ $<5.4$.

dimension when the attractor is projected into $\mathbb{R}^{3}$. Therefore, its self-intersection in $\mathbb{R}^{3}$ has dimension not greater than 1.4, and its measure in $R^{3}$ is therefore zero. This is the region responsible for failure to embed.

Geometric and dynamical tests for embedding involve averaging some real statistic over the entire mapped attractor. That is, they depend upon picking up a signal from a measure zero set. This small signal could easily be obscured by choice of threshold parameters for these two classes of tests. On the other hand, there is no way to misinterpret the zerocrossing signatures of the type shown in Fig. 12.

\section{PARAMETERS}

False near neighbor tests depend on three parameters. One $(\epsilon)$ is used to determine neighbors; a second (5 in the case described above) is used to distinguish between true and false neighbors. The third is a threshold parameter that is used to determine when the fraction of FNN is sufficiently small so that the mapping can be declared an embedding.

Correlation dimension estimates use two parameters. These are lengths: they determine the two ends of the interval over which a linear curve $y=m x+b$ is fit to estimate a slope.

Largest Lyapunov exponent estimates use four input parameters. Two are size estimates. These are sphere radii that are used to determine if points $i$ and $j$ are neighbors. The other two define end points of the forward time interval for which a linear curve $y=m x+b$ is fit in order to estimate the LLE.

Topological tests depend on one parameter. This is a length, used to determine when two points $\mathbf{x}(i)$ and $\mathbf{x}(j)$ are close enough to be considered points on the same unstable periodic orbit, with $j=i+p$, where $p$ is related to the period of the orbit and the time step size $d t$.

In terms of numbers of input parameters required for these types of tests for embeddings, the topological test is the most frugal. 


\section{COMPUTATIONAL LOAD}

Typical scalar data sets consist of $N$ points that sample about $n p$ periods with about $s p p$ points per period, so that $N \simeq n p \times s p p$.

An estimate of the largest Lyapunov exponent requires searching for nearest neighbors. This is asymmetric: if $j$ is a nearest neighbor of $i, i$ is not generally a nearest neighbor of $j$. This requires searching over all point pairs $1 \leqslant i \neq j \leqslant N$, with a computational load of $N(N-1)$.

The FNN and $D_{2}$ estimates involve computing distances, which are symmetric in $i$ and $j$. The search is over $1 \leqslant i<j$ $\leqslant N$, with a computational load of $\frac{1}{2} N(N-1)$.

The number of point pairs in each search can be reduced slightly by blocking out dynamically correlated points: $|i-j|<q$, where $q$ is conveniently taken as about $s p p / 4$.

Topological tests involve close returns searches for unstable periodic orbits. The search is symmetric in $i$ and $j$. Assuming orbits of period up to 6 are sufficient, the search is over $i+\frac{1}{2} s p p<j<\left(6+\frac{1}{2}\right) s p p$, with a computational load of about $(N-6 s p p) \times 6 s p p$. The term quadratic in $s p p$ is due to sample-size edge effects.

No matter the sample size, FNN and $D_{2}$ estimates require about half the computational effort as LLE estimates. Periodic orbit searches require about $6 / n p$ of the computational effort. Useful data sets typically have $n p \simeq 100, s p p \simeq 100$. For such data sets the topological approach requires about $6 \%$ of the computational search effort as LLE estimates.

The classical tests involve symmetric or asymmetric (in the indices $i$ and $j$ ) searches over all point pairs followed by some post-search processing and averaging. Decisions are made by introducing some sort of threshold and deciding whether the average reaches this threshold. The average varies from data set to data set (cf. Fig. 13) and there is no statistical theory to go with any of this (cf. Grassberger and Procaccia [19]), despite our findings (cf. Fig. 14).

By contrast, the topological test involves a symmetric search over all point pairs followed by extraction of specific small subsets of points belonging to structures that are invariant from one data set to another (periodic orbits). There is no averaging. What can differ from one data set to another is how close the minimum gets to zero (cf. Fig. 12), but not the shape of the minimum distance curve or the conclusion of whether a zero crossing occurs or not.

\section{NOISE}

The deleterious effect of noise on estimates of fractal dimensions and Lyapunov exponents has been extensively documented $[49,50]$. In view of the failure of both types of tests to predict correctly when a mapping was an embedding, it was not deemed necessary to determine how much worse they behaved by adding noise to our two test data sets.

Topological tests and analyses are robust and degrade gracefully with respect to noise $[7,11,12]$. The very first studies of the effects of noise on this test were carried out on experimental data $[7,52]$. It has been well documented that the ability to extract unstable periodic orbits from data decreases as the signal-to-noise level decreases, but the most susceptible orbits, the first orbits masked by increasing noise level, are typically the highest period orbits. As the analysis depends more heavily on the lower period orbits, noise is far less inimical to topological analyses than to geometric or dynamical analyses. In this sense, in the domain of topological analysis, "Murphy takes a vacation" $[7,11,12]$.

It can also be argued that estimates of minimum distances (cf. Fig. 12) or linking numbers are corrupted by noise. Noisy periodic orbits are closed orbits. This allows a Fourier representation for each periodic orbit both to force closure as well as filter noise by projecting higher-frequency components to zero [1]. The Fourier representations of orbits can be used in place of the noisy orbits for the minimum distance and linking number calculations. Fourier representations are not available in the same way for geometric or topological analyses since the trajectories being analyzed there are chaotic, not periodic.

Finally, dimension estimates are impossible, and Lyapunov exponent estimates are impractical, for nonstationary data. Successful topological analyses have already been carried out for nonstationary experimental data [51].

\section{CONCLUSIONS}

The two classical types of tests for whether a mapping is an embedding, depending on geometric and dynamical measures, have recently been joined by a third type, depending on topological measures [1]. The latter test is only applicable to three dimensions, while the classical tests do not have such a limitation. However, most claims of embeddings into higher-dimensional spaces stop there: there is little postembedding self-consistency checking. In three dimensions a successful embedding is the beginning of a more detailed analysis stage in which it is possible to determine the mechanism responsible for generating chaotic data $[37,38]$.

For this reason alone it is useful to compare the reliability, simplicity, and robustness of these three different types of tests where they can be compared: on three-dimensional attractors. We have done so in this work. We find that the classical tests are often unable to suggest when mapping parameter values provide an embedding and when they do not; when they have been able to suggest values, they have been wrong in every instance studied here. This result calls into question the reliability of results of the traditional methods in higher dimensions where topological methods do not yet exist to reject or fail to reject the results of traditional tests.

\section{ACKNOWLEDGMENT}

R.G. thanks Centre National de la Recherche Scientifique (CNRS) for support at COmplexe de Recherche Interprofessionnel en Aérothermochimie (CORIA) for 2006-2007. 
[1] C. Letellier, I. M. Moroz, and R. Gilmore (unpublished).

[2] F. Takens, in Proceedings of the Symposium on Dynamical Systems and Turbulence, Warwick, 1979-1980, edited by D. Rand and L. S. Young, Lecture Notes in Mathematics Vol. 898 (Springer-Verlag, Berlin, 1981), p. 366.

[3] R. Mañé, in Proceedings of the Symposium on Dynamical Systems and Turbulence, Warwick, 1979-1980, edited by D. Rand and L. S. Young, Lecture Notes in Mathematics Vol. 898 (Springer-Verlag, Berlin, 1981), p. 230.

[4] N. H. Packard, J. P. Crutchfield, J. D. Farmer, and R. S. Shaw, Phys. Rev. Lett. 45, 712 (1980).

[5] J.-P. Eckmann and D. Ruelle, Rev. Mod. Phys. 57, 617 (1985).

[6] E. Ott, Rev. Mod. Phys. 53, 655 (1981).

[7] G. B. Mindlin, H. G. Solari, M. A. Natiello, X.-J. Hou, and R. Gilmore, J. Nonlinear Sci. 1, 147 (1991).

[8] H. D. I. Abarbanel, R. Brown, J. J. Sidorowich, and L. Tsimring, Rev. Mod. Phys. 65, 1331 (1993).

[9] H. D. I. Abarbanel, Analysis of Observed Chaotic Data (Springer, New York, 1996).

[10] H. Kantz and T. Schreiber, Nonlinear Time Series Analysis (Cambridge University Press, Cambridge, England, 1997).

[11] R. Gilmore, Rev. Mod. Phys. 70, 1455 (1998).

[12] R. Gilmore and M. Lefranc, The Topology of Chaos (John Wiley \& Sons, New York, 2002).

[13] M. P. Chauve and P. LeGal, Physica D 58, 407 (1992).

[14] B. Saltzman, J. Atmos. Sci. 19, 329 (1962).

[15] E. N. Lorenz, J. Atmos. Sci. 20, 130 (1963).

[16] P. Grassberger, Phys. Lett. 97, 227 (1983).

[17] P. Grassberger and I. Procaccia, Phys. Rev. A 28, 2591 (1983).

[18] P. Grassberger, Phys. Lett. 97A (6), 227 (1983).

[19] P. Grassberger and I. Procaccia, Phys. Rev. Lett. 50, 346 (1983).

[20] D. Ruelle, Proc. R. Soc. London, Ser. A 427, 241 (1990).

[21] M. Lefranc, D. Hennequin, and P. Glorieux, Phys. Lett. A 163, 269 (1992).

[22] M. B. Kennel, R. Brown, and H. D. I. Abarbanel, Phys. Rev. A 45, 3403 (1992).

[23] G. Sugihara and R. M. May, Nature (London) 344, 734 (1990).

[24] D. T. Kaplan and L. Glass, Phys. Rev. Lett. 68, 427 (1992).

[25] A. Wolf, J. B. Swift, H. L. Swinney, and J. A. Vastano, Physica D 16, 285 (1985).

[26] S. Sato, M. Sano, and Y. Sawada, Prog. Theor. Phys. 77, 117 (1987).

[27] J. D. Farmer and J. J. Sidorowich, Phys. Rev. Lett. 59, 845 (1987).
[28] R. Stoop and J. Parisi, Physica D 50, 89 (1991).

[29] M. T. Rosenstein, J. J. Collins, and C. J. De Luca, Physica D 65, 117 (1993).

[30] J. Kaplan and J. A. Yorke, in Functional Differential Equations and Approximations of Fixed Points, edited by H.-O. Peitgen and H.-O. Walther, Lecture Notes in Mathematics Vol. 730 (Springer, Berlin, 1979), pp. 228-237.

[31] J.-P. Eckmann, S. O. Kamphorst, D. Ruelle, and S. Ciliberto, Phys. Rev. A 34, 4971 (1986).

[32] U. Parlitz, Int. J. Bifurcation Chaos Appl. Sci. Eng. 2, 155 (1992).

[33] H. G. Solari and R. Gilmore, Phys. Rev. A 37, 3096 (1988).

[34] D. Auerbach, P. Cvitanovic, J.-P. Eckmann, G. Gunaratne, and I. Procaccia, Phys. Rev. Lett. 58, 2387 (1987).

[35] H. Whitney, Ann. Math. 37, 645 (1936).

[36] T. Sauer, M. Casdagli, and J. A. Yorke, J. Stat. Phys. 65, 579 (1991).

[37] N. Romanazzi, M. Lefranc, and R. Gilmore, Phys. Rev. E 75, 066214 (2007).

[38] R. Gilmore, C. Letellier, and N. Romanazzi, J. Phys. A 40, 13291 (2007).

[39] T. Ondarçuhu, G. B. Mindlin, H. L. Mancini, and C. Pérez Garcia, Phys. Rev. Lett. 70, 3892 (1993).

[40] G. B. Mindlin and H. G. Solari, Phys. Rev. E 52, 1497 (1995).

[41] M. Huerta, D. Krmpotic, G. B. Mindlin, H. Mancini, D. Mazza, and C. Perez-Garcia, Physica D 96, 200 (1996).

[42] T. D. Tsankov, A. Nishtala, and R. Gilmore, Phys. Rev. E 69, 056215 (2004).

[43] I. M. Moroz, C. Letellier, and R. Gilmore, Phys. Rev. E 75, 046201 (2007).

[44] K. A. Robbins, Math. Proc. Cambridge Philos. Soc. 82, 309 (1977).

[45] I. M. Moroz, Int. J. Bifurcation Chaos Appl. Sci. Eng. 13, 147 (2003).

[46] I. M. Moroz, Nonlinear Dyn. 41, 191 (2005).

[47] J.-P. Eckmann, S. O. Kamphorst, and D. Ruelle, Europhys. Lett. 5, 973 (1987).

[48] R. Gilmore, Catastrophe Theory for Scientists and Engineers, (Wiley, New York, 1981).

[49] P. Bryant, R. Brown, and H. D. I. Abarbanel, Phys. Rev. Lett. 65, 1523 (1990).

[50] X. Zeng, R. A. Pielke, and R. Eykholt, J. Atmos. Sci. 44, 649 (1992).

[51] A. Amon and M. Lefranc, Phys. Rev. Lett. 92, 094101 (2004).

[52] G. B. Mindlin and R. Gilmore, Physica D 58, 229 (1992). 